\title{
Technical Note: Improved total atmospheric water vapour amount determination from near-infrared filter measurements with sun photometers
}

\author{
F. Mavromatakis ${ }^{1}$, C. A. Gueymard ${ }^{2}$, and Y. Franghiadakis ${ }^{1}$ \\ ${ }^{1}$ Department of Scienses, Technological Education Institute of Crete, Estavromenos, P.O. Box 1939, 71004 Heraklion, Crete, \\ Greece \\ ${ }^{2}$ Solar Consulting Services, P.O. Box 392, Colebrook, NH 03576, USA
}

Received: 14 March 2007 - Published in Atmos. Chem. Phys. Discuss.: 8 May 2007

Revised: 15 August 2007 - Accepted: 9 September 2007 - Published: 12 September 2007

\begin{abstract}
In this work we explore the effect of the contribution of the solar spectrum to the recorded signal in wavelengths outside the typical 940-nm filter's bandwidth. We employ gaussian-shaped filters as well as actual filter transmission curves, mainly AERONET data, to study the implications imposed by the non-zero out-of-band contribution to the coefficients used to derive precipitable water from the measured water vapour band transmittance. Published parameterized transmittance functions are applied to the data to determine the filter coefficients. We also introduce an improved, three-parameter, fitting function that can describe the theoretical data accurately, with significantly less residual effects than with the existing functions. The moderateresolution SMARTS radiative transfer code is used to predict the incident spectrum outside the filter bandpass for different atmospheres, solar geometries and aerosol optical depths. The high-resolution LBLRTM radiative transfer code is used to calculate the water vapour transmittance in the 940-nm band. The absolute level of the out-of-band transmittance has been chosen to range from $10^{-6}$ to $10^{-4}$, and typical response curves of commercially available silicon photodiodes are included into the calculations.

It is shown that if the out-of-band transmittance effect is neglected, as is generally the case, then the derived columnar water vapour is mainly underestimated by a few percents. The actual error depends on the specific out-of-band transmittance, optical air mass of observation and water vapour amount. Further investigations will use experimental data from field campaigns to validate these findings.
\end{abstract}

Correspondence to: F. Mavromatakis

(fotis@stef.teiher.gr)

\section{Introduction}

Water vapour is a key constituent of the atmosphere, particularly in the lower layers of the troposphere. It determines in part cloudiness and rainfall, and therefore needs to be known accurately for meteorological and climatological purposes, including weather forecasts and energy budget studies. Being very variable on daily, intraseasonal (Chen et al., 1996) and seasonal time scales, its measurement has been the subject of continuous improvements over the last decades. Recent studies (Ross and Elliot, 1996; Elliot and Angell, 1997) have shown that an increasing trend in water vapour is discernible on a continental scale, which is of concern because of the interaction between water vapour, atmospheric heating/cooling, and various feedbacks linked to the hydrological cycle as a whole. This observation is of great significance considering the implications of the current global climate change. For this reason, any trend in water vapour must be monitored closely and regionally with appropriate instrumentation.

Radiosonde sites are numerous and provide the longest historical record, but are usually launched only twice a day. Recent ground-based instrumentation include GPS receivers and microwave radiometers, which are gaining acceptance in the community. In some cases, ground-based data are assimilated with spaceborne retrievals to generate gridded datasets (Randel et al., 1996). Sun photometers constitute the only ground-based optical alternative to these measurements. They require a visible sun's disc, which is used both for calibration and radiometric determination of the optical depth of water vapour and of another variable atmospheric constituent, namely the aerosols. Under cloudless skies, aerosols and water vapour are the two major sources of extinction in the shortwave spectrum, and their time variations are generally uncorrelated (Holben, 1990). It is therefore convenient

Published by Copernicus Publications on behalf of the European Geosciences Union. 
that they can be retrieved simultaneously from a single instrument. Some countries developed water vapour measurement networks using a combination of GPS receivers and sun photometers (Bokoye et al., 2003; Morland et al., 2006). International sun photometer networks, such as AERONET (Holben et al., 1998) and the Global Atmosphere Watch ${ }^{1}$ (GAW) also exist for combined aerosol and water vapour measurement, totaling hundreds of sites worldwide. A handy feature of sun photometers is that they are portable, and therefore can be embarked either on terrestrial vehicles for in-situ ground truthing (Bruegge et al., 1990) or regional assessment, or on airborne platforms for profiling or radiative closure experiments (Livingston et al., 2003).

Although various techniques exist to determine water vapour from multiwavelength sun photometers, the most common is based on single-filter measurements in the 940$\mathrm{nm}$ water vapour absorption band, the strongest one below the 1100-nm limit of silicon detectors, and the only available water vapour channel in most sunphotometers. The technique, which has matured over the years (Bruegge et al., 1992; Ingold et al., 2000; Michalsky et al., 1995; Schmid et al., 1996, 2001; Thome et al., 1992), consists in determining experimentally the extinction or "loss of light" due to water vapour absorption. Theoretical models are required to estimate the columnar water vapour responsible for the measured extinction. Refinements to this technique are proposed here, by investigating and quantifying the effects caused by the combination of imperfect filter out-of-band (OOB) rejection, varying solar zenith angle, water vapour and aerosols, and non-zero site elevation, on the derivation of water vapour.

This contribution is aimed at improving the determination of precipitable water by eliminating various sources of systematic or random errors that have been generally overlooked so far.

\section{The method}

Sun photometers use filters, in carefully chosen wavelength bands, in order to determine aerosol optical depths and columnar water vapour. Manufacturers quote, among other characteristics, the blocking of their filters. Ideally, each filter's transmittance should be less than this number for all out-of-band wavelengths listed in its datasheet. Various issues related to the nature of the interference filters have been raised by several authors (e.g. Schmid et al., 1998; Box, 1981; Basher and Matthews, 1977).

Assume that $T_{\mathrm{F}}(\lambda)$ and $T_{\mathrm{D}}(\lambda)$ are the wavelengthdependent responses of the filter and detector used to record the incoming photons, respectively. The incident photons may be of any wavelength, whereas the response of a silicon photodiode is limited to the range $\sim 300-1100 \mathrm{~nm}$. In

\footnotetext{
${ }^{1}$ http://www.wmo.int/web/arep/gaw/gaw_home.html
}

this waveband, water vapour absorption is strongest around 940-nm. The methodology detailed below uses filter measurements in this absorption band to obtain the total water vapour amount in a vertical column that would extend from the ground to the limit of the atmosphere. This amount is usually expressed as the equivalent depth of condensed water, and referred to as precipitable water $(P W)$.

The extraterrestrial solar spectrum is described by a function $I_{0}(\lambda)$, the shortwave range (e.g. $300-4000 \mathrm{~nm}$ ) of which is only of concern here. There exists a number of references in the literature that describe the extraterrestrial solar spectrum. The recent spectrum of Gueymard (2004) is adopted here. It is used in the SMARTS radiative code package ${ }^{2}$ (Gueymard, 2001), and is also available directly from http://rredc.nrel.gov/solar/spectra/am0/special. html\# newgueymard. In what follows, all calculations are limited to the range defined by the optical characteristics of the detector/filter system, including its out-of-band (OOB) contribution.

The average atmospheric transmittance, $T$, weighted by the detector/filter system's response is given by

$$
T=\frac{\int I_{\mathrm{o}}(\lambda) T_{\mathrm{F}}(\lambda) T_{\mathrm{D}}(\lambda) e^{-m \tau_{\lambda}} d \lambda}{\int I_{\mathrm{o}}(\lambda) T_{\mathrm{F}}(\lambda) T_{\mathrm{D}}(\lambda) d \lambda}
$$

where $m$ is the optical air mass and $\tau_{\lambda}$ stands for the total optical depth, including all major sources of extinction (Rayleigh, ozone, mixed gases, trace gases, aerosols and water vapour).

Because of the lack of complete OOB rejection in practice, two areas of integration are defined. The first characterizes the waveband where the small but finite OOB response of the filter contributes to the recorded signal. Since the response of the filter in the range of, e.g., $300-910 \mathrm{~nm}$ and $960-1100 \mathrm{~nm}$ is not zero, it is clear that a low level signal will leak in and contribute to the final one. The actual intensity of this parasitic signal depends upon the OOB blocking. The worse the blocking, the higher its contribution to the recorded signal and the larger the uncertainty in the derived quantities. The second integral covers the waveband where the filter's transmittance is above a certain limit (e.g., $10^{-6}$ or $10^{-4}$ ). Typically, this waveband ranges from 910 to $960 \mathrm{~nm}$. Consequently, we may further process Eq. (1), for the specific water absorption band, as

$$
T=\frac{\int_{\text {in }} I_{\mathrm{o}} T_{\mathrm{F}} T_{\mathrm{D}} e^{-m \tau_{\lambda}} d \lambda+\int_{\text {out }} I_{\mathrm{o}} T_{\mathrm{F}} T_{\mathrm{D}} e^{-m \tau_{\lambda}} d \lambda}{\int_{\text {in }} I_{\mathrm{o}} T_{\mathrm{F}} T_{\mathrm{D}} d \lambda+\int_{\text {out }} I_{\mathrm{o}} T_{\mathrm{F}} T_{\mathrm{D}} d \lambda}
$$

The terms "in" and "out" refer to the inband and OOB integration limits, respectively. Assuming that absorption by gases other than water vapour and ozone is negligible in the waveband $910-960 \mathrm{~nm}$, the left term in the numerator of Eq. (2) can be written as $e^{-m_{\mathrm{R}} \tau_{\mathrm{R}}} e^{-m_{\mathrm{O}_{3}} \tau_{\mathrm{O}_{3}}} e^{-m_{\text {aer }} \tau_{\text {aer }}} \int_{\text {in }} I_{\mathrm{o}} T_{\mathrm{F}} T_{\mathrm{D}} e^{-\tau_{w \lambda}} d \lambda$, while the

\footnotetext{
2 available from http://rredc.nrel.gov/solar/models/SMARTS/
} 
right one can be simplified into $\int_{\text {out }} I T_{\mathrm{F}} T_{\mathrm{D}} d \lambda$. The optical depths $\tau_{\mathrm{R}}, \tau_{\mathrm{O}_{3}}$, and $\tau_{\text {aer }}$ are assumed nearly constant over the filter's bandwidth, so that their value at or near 940-nm can be used as an average. Note that four specific and distinct optical masses $\left(m_{\mathrm{R}}, m_{\mathrm{O}_{3}}, m_{\text {aer }}\right.$, and $\left.m_{\mathrm{w}}\right)$ are now considered for each extintion process, rather than just the more conventional air mass, which was used in Eqs. (1-2) for conciseness.

The integrated water vapour transmittance finally reads,

$$
\begin{aligned}
T_{\mathrm{w}}= & \frac{\int_{\text {in }} I_{\mathrm{o}} T_{\mathrm{F}} T_{\mathrm{D}} e^{-\tau_{w \lambda}} d \lambda}{\int_{\text {in }} I_{\mathrm{o}} T_{\mathrm{F}} T_{\mathrm{D}} d \lambda+\int_{\text {out }} I_{\mathrm{o}} T_{\mathrm{F}} T_{\mathrm{D}} d \lambda} \\
& +\frac{e^{m_{\mathrm{R}} \tau_{\mathrm{R}}} e^{m_{\mathrm{O}_{3}} \tau_{\mathrm{O}_{3}}} e^{m_{\text {aer }} \tau_{\text {aer }}} \int_{\text {out }} I T_{\mathrm{F}} T_{\mathrm{D}} d \lambda}{\int_{\text {in }} I_{\mathrm{O}} T_{\mathrm{F}} T_{\mathrm{D}} d \lambda+\int_{\text {out }} I_{\mathrm{o}} T_{\mathrm{F}} T_{\mathrm{D}} d \lambda}
\end{aligned}
$$

here $T_{\mathrm{W}}=T e^{m_{\mathrm{R}} \tau_{\mathrm{R}}} e^{m_{\mathrm{O}_{3}} \tau_{\mathrm{O}_{3}}} e^{m_{\text {aer }} \tau_{\mathrm{aer}}}$, and $\tau_{w \lambda}$ denotes the spectral water vapour optical depth. The spectral water vapour transmittance is noted $\mathrm{e}^{-\tau_{w \lambda}}$ rather than $\mathrm{e}^{-m_{w} \tau_{w \lambda}}$ to be consistent with previous contributions (e.g. Ingold et al., 2000; Schmid et al., 2001).

The above four integrals are calculated numerically for any combination of atmospheric profile, geometry and aerosol conditions. Thus, for any given OOB transmittance a total of $6($ atmos $) \times 12($ aerosol $) \times 42$ (airmass) $=3024$ data points are calculated and fitted with appropriate statistical software to a simpler function of the main driving variable, $W=m_{\mathrm{w}} \cdot w$, which represents the total water vapour amount integrated along the slant column. We use the fit program "GaussFit", originally designed to perform astrometry on data from the Hubble Space Telescope, as a robust estimator (HST AST, 2001).

All 3024 rows of data can be fitted simultaneously to any transmittance function, or 12 different fits can be performed, each with 252 rows of data. We preferred the second approach because it allows to monitor the change in coefficient values as a function of $\tau_{\text {aer }}$. Whenever fitting coefficients are quoted in the following, they are derived as the mean of the twelve values mentioned above, while the quoted errors are the standard deviations based on these values. Consequently, the errors are mainly coupled to the variation in the aerosol contribution due to the OOB leakage rather than to the goodness of the fit. When the OOB response is ideally zero, the errors are indeed representative of the goodness of the fit. The error analysis that has been done considers that the absolute error in each individual optical depth is 0.01 . This may be achieved in practice for Rayleigh scattering and ozone absorption. For aerosol extinction however, this is a rather conservative estimate, if we keep into account the sources that may contribute to it (e.g. Shaw, 1976; Schmid and Wehrli, 1995; Reagan et al., 1986; Schmid et al., 1998). Larger errors would simply improve the $\chi^{2}$ values even more.

For any of the ideal atmospheres mentioned in Table 1 , the water vapour transmittance is calculated with the high-resolution (line-by-line) radiative transfer code LBLRTM $^{3}$ v10.3, while the absorbed solar spectrum $I(\lambda)$,

\footnotetext{
${ }^{3}$ http://www.rtweb.aer.com/
}

Table 1. Major parameters and their range.

\begin{tabular}{cl}
\hline \multicolumn{1}{c}{ Parameter } & Value (range) \\
\hline Atmospheres & MLS, MLW, USSA \\
& TRO, SAS, SAW \\
Solar Zenith angle & $0^{\circ}, 82^{\circ}\left(2^{\circ}\right)$ \\
Aerosol turbidity $\beta$ & $0.015-0.405$ \\
OOB & $1,5,10,25$, \\
transmittance $\left(\times 10^{-6}\right)$ & $50,75,100$ \\
\hline
\end{tabular}

outside the filter's bandpass, as well as the optical depths due to Rayleigh, ozone and aerosol extinction are calculated with the moderate-resolution code SMARTS v2.9.5. The LBLRTM code utilizes the HITRAN 2000 spectroscopic database with certain updates from 04/2001 for calculations in the water vapour band around $940-\mathrm{nm}$, as well as "mt_ckd v1.3" for the continuum data (Mlawer et al., 2003). The $\mathrm{RFM}^{4}$ code has also been used to study the OOB effect. This code was originally developed at the department of Atmospheric, Oceanic and Planetary Physics of the University of Oxford. The resulting filter coefficients are close and qualitatively similar to those obtained with LBLRTM, with a typical difference of less than $3 \%$. In the following, the quoted results are based on the LBLRTM code. Our calculations assume a flat OOB filter response with the following possible values in units of $10^{-6}: 0,1,5,10,25,50,75$, and 100 .

The ideal spectral irradiance incident on the filter is calculated for zenith angles ranging from 0 to 82 degrees, in 2 -degree steps. The water vapour optical mass $\left(m_{\mathrm{w}}\right)$, as well as all the other optical masses, is calculated by SMARTS as a function of the sun's zenith angle. The atmosphere models adopted in the calculations are the midlatitude summer (MLS), midlatitude winter (MLW), U.S. Standard Atmosphere (USSA), tropical (TRO), sub-arctic summer (SAS) and sub-artic winter (SAW). The aerosol properties are defined by a Rural aerosol model (Shettle and Fenn, 1979), which is one of the default models implemented in SMARTS. The aerosol optical depth at 1000-nm (also known as the Ångström's turbidity coefficient $\beta$, a conventional measure of aerosol extinction), is varied logarithmically from 0.015 to 0.405 in 12 steps. The adopted conditions are summarized in Table 1.

\section{Results}

\subsection{Gaussian filters}

The above method is implemented first for a gaussian filter centered at $940-\mathrm{nm}$ and characterized by a full width at half maximum (FWHM) of 10-nm. The resulting transmittances

\footnotetext{
${ }^{4}$ http://www-atm.physics.ox.ac.uk/RFM
} 

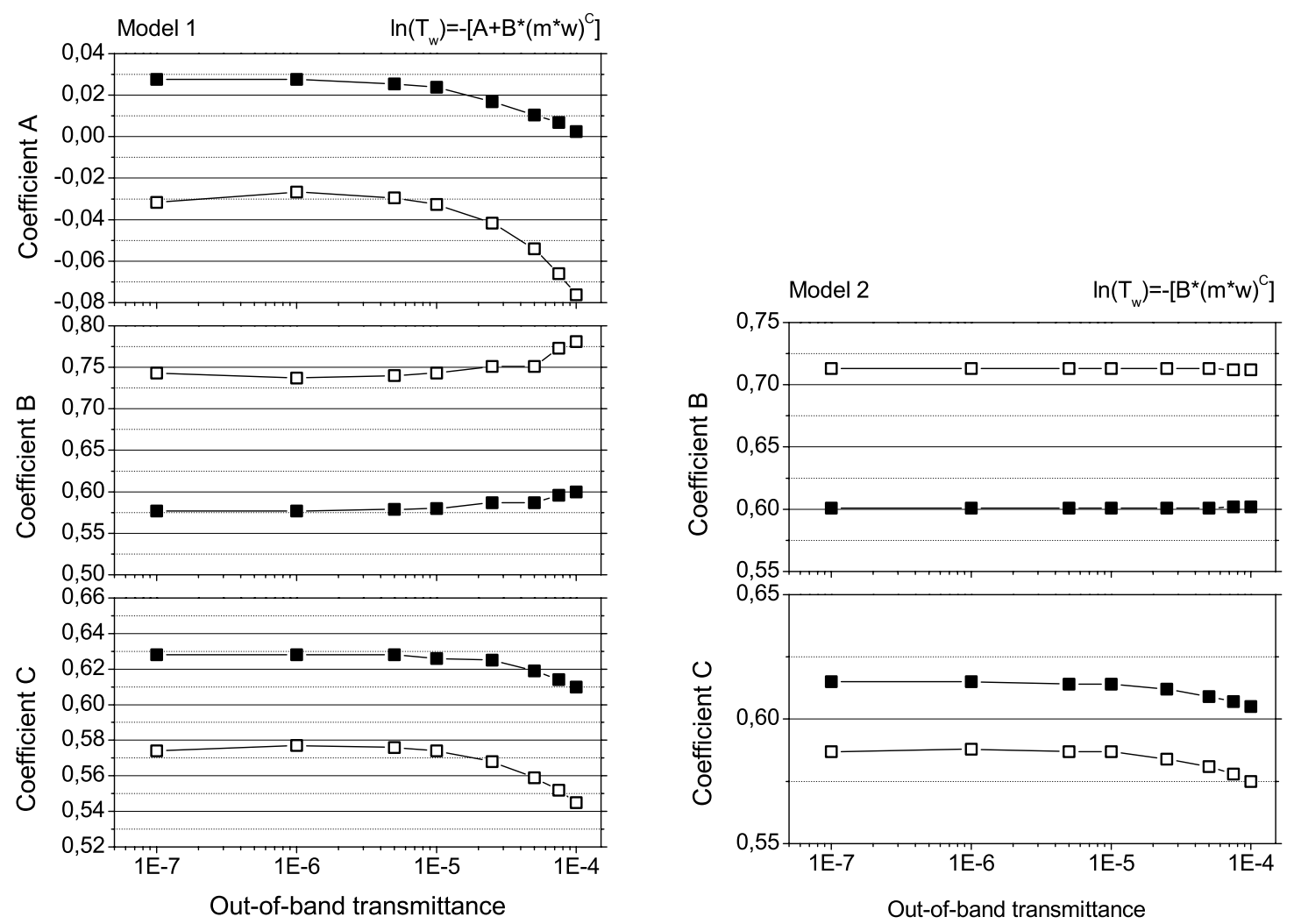

Fig. 1. The filter coefficients $A, B$, and $C$ of Model 1 and $B$ and $C$ of Model 2 are plotted as a function of the out-of-band transmittance. The coefficients for ideal gaussian and AERONET filters are assigned an out-of-band transmittance of $10^{-7}$ instead of zero in order to use a logarithmic scale in the x-axis. The filled squares mark the development of the coefficients in the case of an ideal gaussian filter, while the open squares mark those of an ideally non-leaking bandpass filter used by AERONET in Crete, Greece.

(actually the negative part of their natural logarithm) are fitted with functions of the form " $A+B \cdot W^{\mathrm{C}}$ " (Model 1) and " $B \cdot W^{\mathrm{C}}$ " (Model 2). Model 1 is a modified version of the function proposed by Thome et al. (1992) and similar to that used by (Ingold et al., 2000), while Model 2 is the simpler function most commonly used (e.g. Bruegge et al., 1992; Schmid et al., 2001). Figure 1 shows the variation of coefficients $A, B$, and $C$ for Model 1 (left) and coefficients $B$ and $C$ for Model 2 (right) as a function of the OOB response. The coefficients of the parametrized transmittance for the ideal case are assigned, arbitrarily, an OOB response of $10^{-7}$ in order to display the plot in logarithmic scale.

Note that the derived filter coefficients slightly depend upon the atmospheric code used to perform the appropriate calculations (e.g., Ingold et al., 2000). Our own results, obtained with both the LBLRTM and RFM codes for the same exact atmospheric conditions, support this finding.

The same procedure is repeated for a filter with a FWHM of 15-nm. Broadband filters like this hypothetical one allow for stronger signals to be recorded than with 10-nm FWHM filters, and, consequently, are less affected by the OOB con- tribution. Our numerous simulations show that filters with FWHM narrower than 10-nm are increasingly affected by the OOB effect. The latter is therefore anticorrelated with FWHM.

\subsection{Aerosol Robotic Network filters}

Apart from ideal gaussian filters, we also consider data from typical filters used in the AErosol RObotic NETwork (AERONET; Holben et al., 1998). Two filter transmittance curves have been made available to us. One of these filters was used recently in Crete, Greece at the FORTH site. The second filter is representative of those used at AERONET sites in the continental U.S. We first apply the proposed scheme to the filter used in Crete with Model 2, which is the currently accepted model by AERONET. For an ideal OOB transmittance of zero, we obtain $B=0.713$ and $C=0.587$. For the same filter, AERONET uses $B=0.714$ and $C=0.600$ (A. Smirnov, personal communication, 2007). The agreement is quite fair given the fact that different codes are involved into the calculations and that the adopted atmospheric, geometric, etc. conditions also differ. 
Table 2. Model coefficients for an AERONET filter.

\begin{tabular}{clllll}
\hline \multicolumn{3}{c}{ Model 1 } & \multicolumn{5}{l}{ Model 2 } \\
\hline Out-of-band & A & B & C & B & C \\
\hline 0 & -0.0317 & 0.743 & 0.574 & 0.713 & 0.587 \\
\hline $1 \times 10^{-6}$ & -0.0267 & 0.737 & 0.577 & 0.713 & 0.588 \\
$5 \times 10^{-6}$ & -0.0294 & 0.740 & 0.576 & 0.713 & 0.587 \\
$10 \times 10^{-6}$ & -0.0327 & 0.743 & 0.574 & 0.713 & 0.587 \\
$25 \times 10^{-6}$ & -0.0416 & 0.751 & 0.568 & 0.713 & 0.584 \\
$50 \times 10^{-6}$ & -0.0542 & 0.762 & 0.559 & 0.712 & 0.581 \\
$75 \times 10^{-6}$ & -0.0660 & 0.773 & 0.552 & 0.712 & 0.578 \\
$100 \times 10^{-6}$ & -0.0762 & 0.781 & 0.545 & 0.712 & 0.575 \\
\hline
\end{tabular}

In Fig. 1 the variations of the coefficients for Models 1 and 2 is shown as a function of OOB contamination, while the detailed results are listed in Table 2.

The coefficients obtained with Model 2 for the filter used in the continental USA are $B=0.713$ and $C=0.586$. These values are again close to what AERONET actually uses, $B=0.714$ and $C=0.599$ (A. Smirnov, personal communication, 2007).

The coefficients $B$ and $C$ of Model 1 in Table 2 vary by $\sim 6 \%$ over the range of the considered OOB responses. Coefficient $A$ appears to vary by almost a factor of 3 but what is actually more important is $\mathrm{e}^{-A}$ since the transmittance is given by $T_{w}=\mathrm{e}^{-\left(A+B * W^{C}\right)}$. In this case, the factor $\mathrm{e}^{-A}$ varies by $\sim 5 \%$, a percentage similar to those for coefficients $B$ and $C$. The parameters $A, B$, and $C$ of Model 1 vary more than parameters $B$ and $C$ of Model 2 since the statistical software is able to make the parameters vary more freely due to the additional degree of freedom of Model 1 . The parameter $B$ in Model 2 changes little since it basically determines the transmittance at $W=1$ where the OOB effect is not as strong. Figure 2 shows that this OOB effect influences the optical depth mainly as $W$ increases. In addition, the overall shape of the curve relating the total optical depth and $W$ does not change substantially. This leads to a variation in coefficient $C$ of less than $3 \%$ (Fig. 2). The analysis shows that the two free parameters of Model 2 cannot accomodate for the OOB effect as it becomes stronger and thus, the fits with this model become worse. Consequently, the smaller variations in parameters $B$ and $C$ of Model 2 do not imply that it is a better model than Model 1.

\subsection{An SPM-2000 filter}

Schmid et al. (1996) used the 940-nm channel of sunphotometers to retrieve the columnar water vapour in parallel with other determinations from co-located radiosonde and microwave radiometer equipment. B. Schmid (personal communication, 2007) kindly provided us with the spectral re-

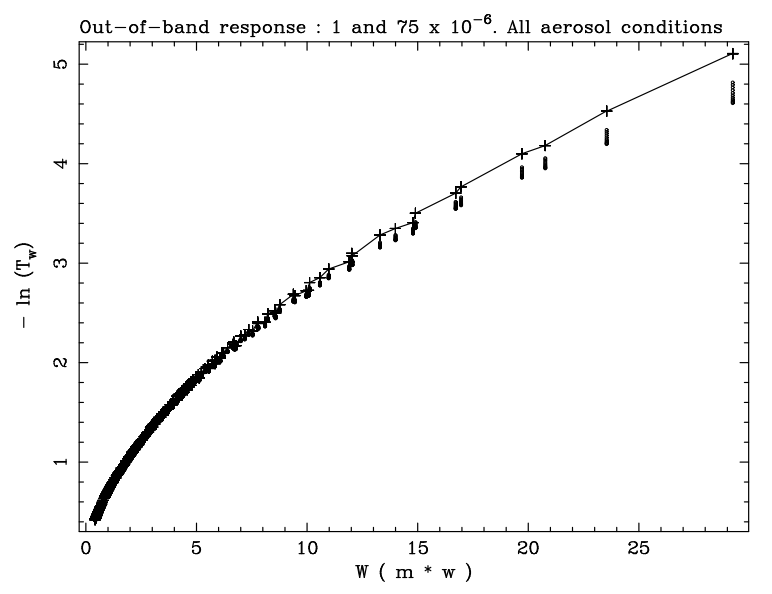

Fig. 2. Total water vapour optical depth as a function of $W$ for OOB transmittances of 1 and $75 \times 10^{-6}$ (plus signs and dots, respectively). The OOB effect is obvious at high $W$.

sponse curve of the filter used in their 1996 paper, as shown in their Fig. 1. We were able to determine the coefficients as $B=0.576$ and $C=0.625$, while Schmid et al. (1996) had found $B=0.549$ and $C=0.629$ with the FASCOD3P code. The agreement is satisfactory considering the different atmospheric codes and the different molecular absorption data involved in the calculations.

For the site in Bern, Schmid et al. (1996) determined the water vapour content independently and established the filter coefficients experimentally. Note that the latter determinations differ from those of the radiative transfer codes, although the statistical significance is questionable $(<4 \sigma$ in $B$ and $<2 \sigma$ in $C$ ). Unfortunately, the OOB response of this filter is not known and we cannot quantitatively estimate its contribution. Nevertheless, we tried several different OOB responses and it can be shown that coefficient $C$ does decrease with increasing OOB level as observed in Fig. 1. 
Table 3. Dependence of model coefficients on atmospheric profile and total water vapour amount for Model 2.

\begin{tabular}{cccc}
\hline Atmosphere & w & Model 2 & \\
& & B & C \\
\hline SAW & 0.416 & 0.704 & 0.586 \\
MLW & 0.852 & 0.714 & 0.584 \\
USSA & 1.416 & 0.710 & 0.584 \\
SAS & 2.081 & 0.715 & 0.581 \\
MLS & 2.922 & 0.726 & 0.579 \\
TRO & 4.115 & 0.740 & 0.574 \\
\hline
\end{tabular}

However, coefficient $B$ also decreases instead of increasing (their Table 1; Schmid et al., 1996). Thus, in the absence of a well-determined OOB response it is not easy to estimate the extent to which the differences in the filter coefficients could be attributed to the OOB effect.

\subsection{Variance in filter coefficients}

The filter coefficients reported up to now are based on data from all model atmospheres (e.g., Table 1). We have determined the corresponding coefficients separately for six model atmospheres using the AERONET filter deployed in Crete, Greece assuming an OOB response of zero. It is found that these coefficients are correlated with the total water vapour amount of the atmosphere (Table 3 ). This fact suggests that the current parametrizations (i.e., Models 1 and 2) need to be improved in order to describe adequately the water vapour transmittance for all types of atmospheric profiles. From Table 3 the resulting variations in the water vapour estimates are of the order of a few percents (less than $\sim 3 \%$ ).

The effect of the observing altitude upon the filter coefficients has also been explored. As in the calculations just above, a perfect bandpass filter (AERONET filter used in Crete, Greece) was adopted and the site altitude was varied from sea level up to $3.5 \mathrm{~km}$, every $0.5 \mathrm{~km}$ (Table 4). Altitudes of 0.1 and $0.3 \mathrm{~km}$ were also considered since a large number of sites are located at altitudes in this range. Additional calculations were performed for two special cases, namely the Bern $(560 \mathrm{~m})$ and Jungfraujoch $(3580 \mathrm{~m})$ sites. Ingold et al. (2000) published filter coefficients based on various radiative transfer codes for these two sites and a relative comparison is desirable. Indeed, the relative variations in the coefficients $A, B$ and $C$ of Model $1(1 \%, 14 \%, 5 \%)$ between the low and high altitude sites of Bern and Jungfraujoch are very similar to those seen in the data presented by Ingold et al. (2000).

\section{Discussion}

The effect introduced by the finite OOB response of filters used in sun photometers is explored in this work along with

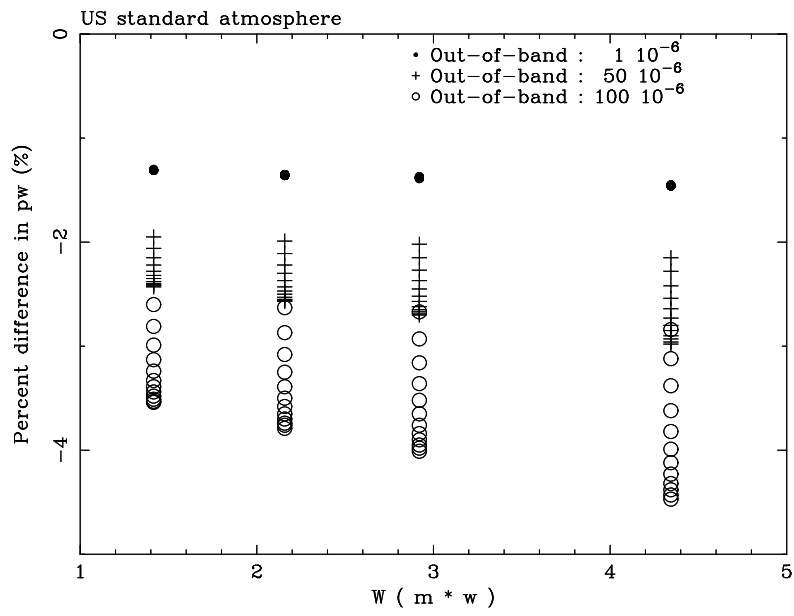

Fig. 3. The U.S. standard atmosphere is invoked to simulate a signal assumed to be sensed by filters of different out-of-band transmittances. The water vapour content is estimated through Model 2, whose coefficients are calculated for an ideal filter (OOB transmittance equal to zero). In this case, PW is systematically underestimated. The different aerosol optical depths used in the calculations show up at different positions of the same symbol at any $x$-value.

different parametrizations of the water vapour transmittance. The OOB response may be small, of the order of $10^{-4}$ or less, but the wavelength integration extends over a large interval $(>700-\mathrm{nm})$, whereas the far stronger in-band response extends over only a few nanometers. In addition, different radiative transfer models predict slightly different optical depths, which in turn interferes with the relationship between $T_{\mathrm{w}}$ and $W$.

A possible way to visualize the differences in the calculated $T_{\mathrm{w}}$ introduced by the OOB response of a filter is to simulate the atmospheric transmittance for known PW conditions and then attempt to retrieve PW with the parametrized functions of a virtual filter. Note that the transmittances are calculated here by taking into account the OOB contribution, whereas the parametrized function only assumes the coefficients for zero OOB response. Figure 3 shows the percentage difference in the determination of PW for USSA and for optical masses of 1, 1.5, 2.0, and 3.0. Under these conditions, $\mathrm{PW}$ is underestimated with respect to the exact water vapour content of USSA $(1.416 \mathrm{~cm})$. The effect is small but systematic. Under real conditions, the actual differences might be smaller since the coefficients reported in Table 2 are based on data from all six atmospheres listed in Table 1. If we only use USSA to determine the filter coefficients, the agreement is improved but the effect is still present, and becomes even worse when retrieving PW for other atmospheric profiles. This approach assumes that the device's signal at the top of the atmosphere can be calculated theoretically, which is not the actual case. 
Table 4. Dependence of model coefficients on site altitude for the AERONET filter used at the FORTH site.

\begin{tabular}{lllllllll}
\hline Altitude $(\mathrm{km})$ & Model 1 & & \multicolumn{5}{c}{ Model 2 } & Model 3 \\
& A & B & C & B & C & A & B & C \\
\hline 0.00 & -0.0317 & 0.743 & 0.574 & 0.713 & 0.587 & 0.0065 & 0.706 & 0.579 \\
0.10 & -0.0338 & 0.742 & 0.572 & 0.711 & 0.585 & 0.0066 & 0.703 & 0.578 \\
0.30 & -0.0252 & 0.727 & 0.575 & 0.704 & 0.585 & 0.0065 & 0.696 & 0.579 \\
0.50 & -0.0138 & 0.709 & 0.578 & 0.696 & 0.584 & 0.0068 & 0.688 & 0.578 \\
0.56 & -0.0171 & 0.712 & 0.577 & 0.695 & 0.585 & 0.0065 & 0.688 & 0.579 \\
1.00 & -0.0038 & 0.683 & 0.582 & 0.680 & 0.584 & 0.0080 & 0.671 & 0.578 \\
1.50 & -0.0055 & 0.671 & 0.581 & 0.665 & 0.584 & 0.0095 & 0.657 & 0.579 \\
2.00 & -0.0143 & 0.667 & 0.572 & 0.652 & 0.581 & 0.0045 & 0.648 & 0.579 \\
2.50 & -0.0162 & 0.653 & 0.566 & 0.635 & 0.578 & -0.0020 & 0.637 & 0.579 \\
3.00 & -0.0162 & 0.634 & 0.563 & 0.617 & 0.577 & 0.0021 & 0.616 & 0.577 \\
3.50 & -0.0231 & 0.626 & 0.553 & 0.601 & 0.578 & -0.0024 & 0.602 & 0.579 \\
3.58 & -0.0246 & 0.625 & 0.551 & 0.598 & 0.578 & -0.0066 & 0.601 & 0.579 \\
\hline
\end{tabular}

The common practice is the utilization of "modified Langley plots" to determine the "air-mass zero" (AM0) voltage $\left(V_{\mathrm{o}}\right)$, where the logarithm of the observed voltage $(V)$ is plotted against $m_{\mathrm{w}}^{\mathrm{C}}$ (Eq. 4), according to

$$
\ln (V)=\ln \left(V_{\mathrm{o}}\right)-\left(A+B\left(m_{\mathrm{w}} \cdot w\right)^{C}\right) .
$$

Equation (4) is descriptive of the use of either Model 1 if $A \neq 0$ or Model 2 if $A=0$. Since coefficient $C$ decreases with increasing OOB response (Table 2), it is clear that the correct $\mathrm{X}$-values will consequently be lowered. Thus, the corresponding AM0 voltage will be higher than the voltage that would be determined assuming a perfect bandpass filter. Given this condition and the fact that the recorded voltage is independent of the filter coefficients, then it can be shown through Eq. (4) that

$w<\frac{1}{m_{\mathrm{w}}}\left[\frac{A^{\prime}-A+B^{\prime}\left(m_{\mathrm{w}} w_{\mathrm{o}}\right)^{\mathrm{C}^{\prime}}}{B}\right]^{\frac{1}{C}}$

where only the coefficients marked with a prime incorporate the out-of-band contribution. It is assumed that the best estimate of the precipitable water, $w_{\mathrm{o}}$, will be given by the parametrized function with these coefficients (prime sign). Equation (5) can be simplified in the case of Model 2 to

$\frac{w}{w_{\mathrm{o}}}<\left(m_{\mathrm{w}} w_{\mathrm{o}}\right)^{\mathrm{C}^{\prime} / \mathrm{C}-1}$

since $A^{\prime}=A=0$ and $B^{\prime} \simeq B$ (Table 2). This equation shows that the water vapour amount is underestimated by $1-3 \%$ for OOB responses in the range of $25 \times 10^{-6}$ to $100 \times 10^{-6}$. The data reduction shows that if the OOB response increases, the goodness of the fits for Model 1 is still acceptable, whereas the fits get worse for Model 2. In the case of an OOB response of $10 \times 10^{-6}$ or less, this effect is not significant. Thus, it is concluded that high OOB responses should be taken into account in the filter coefficient determination in an attempt to minimize systematic errors.

As noted in Sect. 3.4 the coefficients show a clear dependence on the integrated water vapour amount of the atmospheric profile adopted in the calculations with the AERONET data. It is therefore desirable to use a parametrized function that would accurately describe PW amounts from the largest possible number of different atmospheres. Here, we propose to combine Model 1 and Model 2 into a new function (Model 3) of the form

$T_{w}=e^{-\left[A \cdot w \cdot\left(m_{\mathrm{w}} \cdot w\right)^{C}+B \cdot\left(m_{\mathrm{w}} \cdot w\right)^{C}\right]}=e^{-(A \cdot w+B) \cdot\left(m_{\mathrm{w}} \cdot w\right)^{C}}$.

As will be discussed in what follows, Model 3 can describe the water vapour transmittance better than Model 1 or Model 2, presumably for any atmospheric profile and for a wide range of water vapour and air mass values (through $W)$. An advantage of this function over Model 1 is that if $w \rightarrow 0$, then $T_{w} \rightarrow 1$, whereas Model 1 , in this case, predicts a constant offset $\left(\mathrm{e}^{-A}\right)$. In addition, it allows for the calibration of the zero air mass voltage through modified Langley plots without modification. This is stressed because more complicated functions could be developed to better fit the data, but then would not lend themselves to a linear relation between the logarithm of the observed voltage and the power of the water vapour optical mass.

Figure 4 shows the difference in water vapour transmittance between the predictions of Models 1, 2 and 3 and the line-by-line calculations, and for a number of atmospheric profiles. Generally, Model 3 performs better than the other models in different atmospheric environments. The advantage of this model is that a single set of coefficients can be used to represent very different environmental conditions. The calculated uncertainty in the retrieved $w$ caused solely by imperfections in the fitting model varies as a function of w, typically, between about $-4 \%$ and $+7 \%$ for Models 1 and 2, 

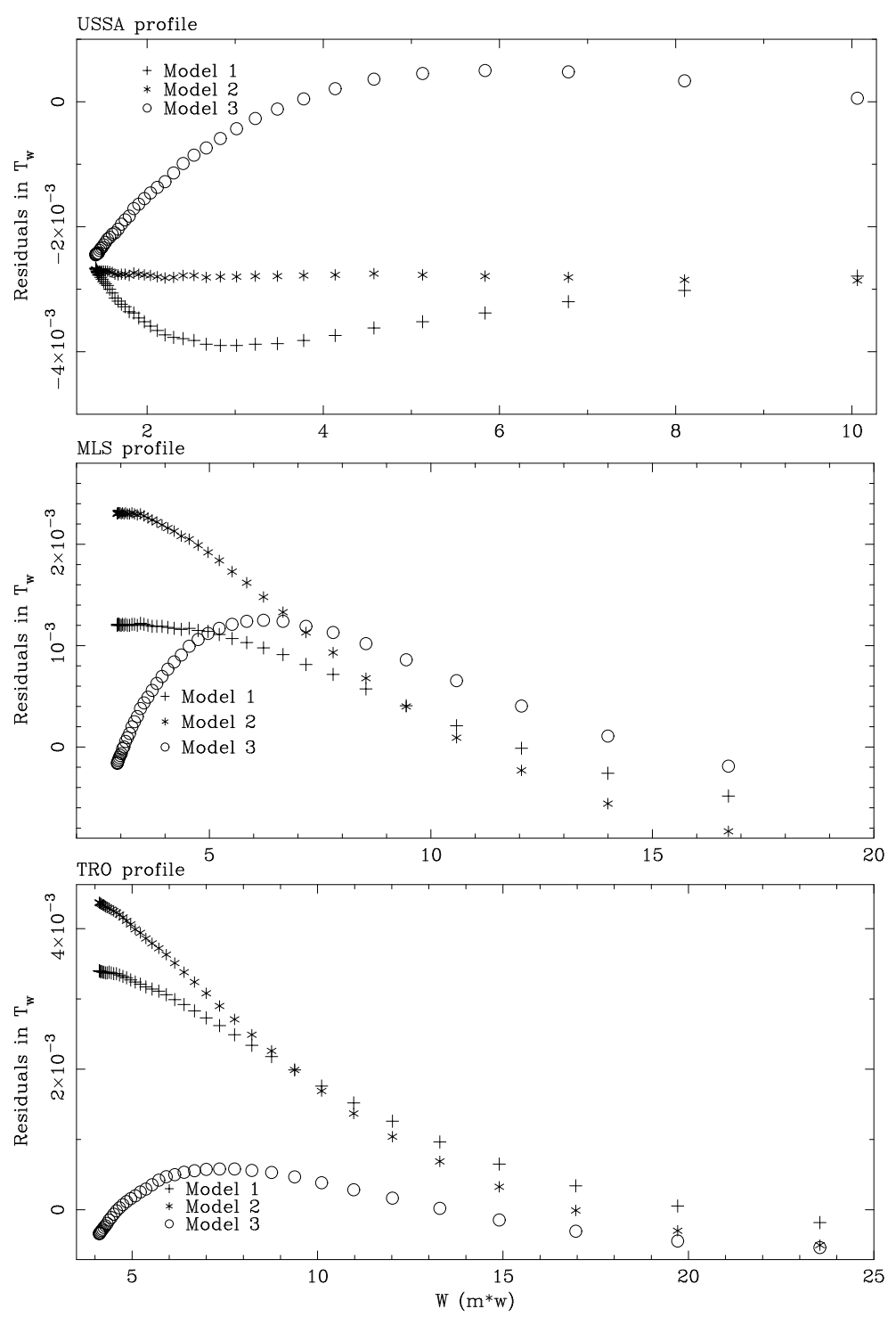

Fig. 4. Residual water vapour transmittance $\left(T_{\text {model }}-T_{\text {simul }}\right)$ as a function of slant water vapour, obtained with three different atmospheric profiles. The simulated data are created under the assumptions appearing in Table 1. The proposed model (Model 3) produces a lower residual than existing Models 1 and 2 over a large range of water vapour conditions.

compared to $-3 \%$ and $+3 \%$ for Model 3 (Fig. 5). These uncertainties increase significantly when adding the effects due to typical OOB (e.g., 10 ${ }^{-4}$ ) and lack of altitude correction.

As a further test, we simulated fixed amounts of PW based on all atmospheric profiles reported in Table 1, using values differing widely from the default one. The selected PW values were $0.5,1.0,2.0,3.0,4.0$, and $5.0 \mathrm{~cm}$. The simulated data points were also limited in air mass $(<4)$ and in transmittance $(>5 \%)$, whichever condition was true. This resulted in more than 1300 data points that could be used to retrieve PW with the aid of Models 1, 2 and 3. The solutions of Models 1 and 2 are algebraic, while that of Model 3 is numerical, given its specific functional form. The coefficients used in these models were determined using all atmospheric profiles with their default values as stated in Table 1.

In Fig. 5 we show the percentage difference between the retrieved and the true PW as a function of the latter. It is evident that Model 3 performs better over the full range of the simulated PW values. Models 1 and 2 show a smaller degree of uniformity in retrieving $P W$ over the same range. This is an important point because determing the filter coefficients for a certain atmospheric profile, e.g., the USSA profile, does not imply that the retrieved PW will be correct over all its full observable range. AERONET Level 2.0 data show that there exist sites that experience yearly variations in PW of an order of magnitude or more (e.g., the GSFC site). In such 


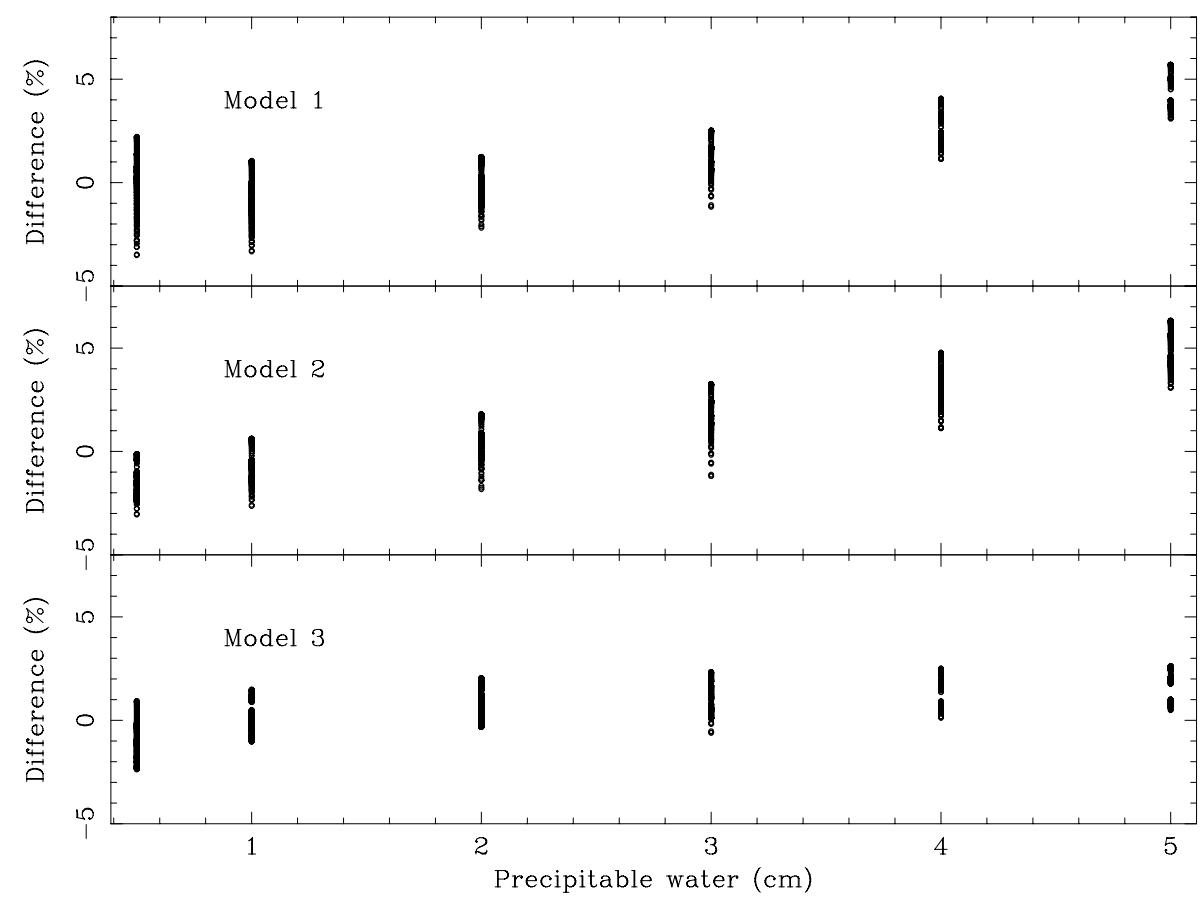

Fig. 5. Uncertainty in the retrieved PW with three possible models. The difference between the retrieved and the actual PW is shown for all three models as a function of the true PW. Model 3 shows a relatively stable behaviour over the whole range of PW values used in this study. In addition, it displays the smallest scatter as a function of air mass, for any given PW.

cases, a model is needed to provide correct estimates of PW from $\sim 0.5 \mathrm{~cm}$ to $5.0 \mathrm{~cm}$ and Fig. 5 shows that for any given $\mathrm{PW}$, Model 3 provides the smallest scatter as a function of varying air mass or atmospheric profile.

Results displayed in Table 4 imply that altitude can have an impact of about $2 \%$ on the estimated PW at low-altitude sites, e.g., for a site located at $300 \mathrm{~m}$ that would be using coefficients calculated for zero elevation. For a high altitude site (e.g. $3000 \mathrm{~m}$ ), this impact increases to $20 \%$. It is evident that altitude is a non-negligible factor, but is usually neglected in practise. Furthermore, the last column of Table 4 shows a unique feature of Model 3: the exponent of $\mathrm{W}$ is essentially independent of altitude. As it is also independent from the water vapour profile and from the total PW, it is therefore determined completely by the water vapour $a b-$ sorption features and by the filter's optical characteristics.

Although all the individual effects considered above may be of the order of only a few percents in most cases, it is argued that they should be properly taken care of to avoid systematic errors. The magnitude of the OOB effect on PW obviously depends on the filter's optical characteristics, which must be measured precisely. Accuracy issues related to the hardware design and implementation constitute a different topic, which is beyond the scope of this work. An experimental validation of the proposed model, using results from field campaigns with sun photometers, microwave radiometers, GPS sensors and radiosonde devices is currently in development.
The OOB effect is expected to affect aerosol channels also, since calibration measurements usually take place at high altitude sites where aerosol effects are minimized, while the actual measurements take place at low altitude sites. Aerosols are known to display a strong wavelength-dependent optical depth behavior causing sunlight to be more depleted at blue wavelengths than at red wavelengths. This effect is significantly suppressed at the calibration sites. For example, the aerosol optical depth at $675-\mathrm{nm}$ at Mauna Loa is typically below 0.01, while e.g. at the FORTH site in Crete values are around $0.15-0.17$. The OOB influence will not simply cancel out when determing $\tau=\frac{1}{m} \ln \left(\frac{V o}{V}\right)$, and further work is required to study this aspect of the OOB effect.

\section{Conclusions}

The method of determining the water vapour with sun photometers is well proven, but there are certain issues that may introduce small, possibly systematic errors in the estimation of PW under realistic conditions. Our simulations show that the out-of-band contribution may have a non-negligible impact on the retrieved PW. Therefore, filters in the 940$\mathrm{nm}$ band should have an OOB rejection of $10^{-5}$ or better. In addition, the observing site's altitude is found to significantly affect the coefficients used to model the water vapour transmittance as a function of PW. These coefficients should 
therefore be calculated for each individual filter (depending on its transmittance and OOB rejection) and location of use.

The residuals of the parametrized transmittances show that the current functions (Models 1 and 2) cannot compensate for the different amounts of water vapour existing in various model atmospheres. A new model with three coefficients (Model 3) is therefore proposed. It is found to perform better (with reduced bias) than the existing models when subjected to widely varying atmospheric profiles. It is associated with a constant PW exponent for any altitude and is also characterised by a small scatter, conducive of reduced random errors, even at large slant column water vapour amounts. Model 1 performs better than Model 2 in terms of correctly estimating PW. However, both models tend to overestimate $\mathrm{PW}$, by up to $5 \%$, at high PW values $(\geq 4 \mathrm{~cm})$. Under dry conditions $(w<1 \mathrm{~cm})$, Models 1 and 2 underestimate PW by just a few percents. In addition to its significant advantages, our analysis shows that Model 3 provides more accurate estimates of PW (by $2 \%$ on the average) over a wide range of water vapour amounts.

Acknowledgements. The authors would like to thank A. Smirnov and B. Schmid for supplying the digital data of the filters used in the calculations. We also thank the referees for their comments, which helped us clarify certain issues of this work.

Edited by: A. Hofzumahaus

\section{References}

Alexandrov, M., Cairns, B., Lacis, A. A., and Carlson, B. E.: New Developments in Multi-Filter Rotating Shadowband Radiometer Data Analysis, 16th ARM Science Team Meeting Proceedings, Albuquerque, NM (available from http://www.arm.gov/ publications/proceedings.stm), 2006.

Basher, R. E. and Matthews, W. A.: Problems in the use of interference filters for spectrophotometric determination of total ozone, J. Appl. Meteorol., 16, 795-802, 1977.

Bokoye, A. I., Royer, A., O’Neill, N. T., Cliche, P., McArthur, L. J. B., Teillet, P. M., Fedosejevs, G., and Theriault, J. M.: Multisensor analysis of integrated atmospheric water vapour over Canada and Alaska. J. Geophys. Res., 108, D15, doi:10.1029/2002JD002721, 2003.

Box, M. A.: Finite bandwidth and scattered light effects on the radiometric determination of atmospheric turbidity and the solar constant, Appl. Optics, 20(13), 2215-2219, 1981.

Bruegge, C. J., Conel, J. E., Margolis, J. S., Green, R. O., Toon, G., Carrere, V., Holm, R. G., and Hoover, G.: In-situ atmospheric water-vapor retrieval in support of AVIRIS validation, Proc. SPIE \#1298, 150-163, 1990.

Bruegge, C. J., Conel, J. E., Green, R. O., Margolis, J. S., Holm, R. G., and Toon, G.: Water vapour column abundance retrievals during FIFE, J. Geophys. Res., 97(D17), 18 759-18 768, 1992.

Chen, T. C., Pfaendtner, J., Chen, J. M., and Wikle, C. K.: Variability of the global precipitable water with a timescale of 90-150 days, J. Geophys. Res., 101(D5), 9323-9332, 1996.
Elliott, W. P. and Angell, J. K.: Variations of cloudiness, precipitable water, and relative humidity over the United States: 19731993, Geophys. Res. Lett., 24, 41-44, 1997.

Gueymard, C.: Parametrized transmittance model for direct beam and circumsolar spectral irradiance, Solar Energy, 71, 325-346, 2001.

Gueymard, C.: The sun's total and spectral irradiance for solar energy applications and solar radiation models, Solar Energy, 76, 423-453, 2004.

Holben, B. N. and Eck, T. F.: Precipitable water in the Sahel measured using sun photometry, Agr. For. Meteorol., 52, 95-107, 1990.

Holben, B. N., Eck, T. F., Slutsker, I., Tanré, D., Buis, J. P., Vermote, E., Reagan, J. A., Kaufman, Y. J., Nakajima, T., Lavenu, F., Jankowiak, I., and Smirnov, A.: AERONETNA federated instrument network and data archive for aerosol characterization, Remote Sens. Environ., 66, 1-16, 1998.

HST Astrometry Science Team: http://clyde.as.utexas.edu, 2001.

Ingold, T., Schmid, B., Mätzler, Demoulin, P., and Kämpfer, N.: Modeled and empirical approaches for retrieving columnar water vapour from solar transmittance measurements in the $0.72,0.82$, and $0.94 \mu \mathrm{m}$ absorption bands, J. Geophys. Res., 105(D19), 24 327-24 343, 2000.

Livingston, J. M., Russell, P. B., Reid, J. S., Redemann, J., Schmid, B., Allen, D. A., Tores, O., Levy, R. C., Remer, L. A., Holben, B. N., Smirnov, A., Dubovik, O., Welton, E. J., Campbell. J. R., Wang, J., and Christopher, S. A.: Airborne sun photometer measurements of aerosol optical depth and columnar water vapor during the Puerto Rico Dust Experiment and comparison with land, aircraft, and satellite measurements, J. Geophys. Res., 108, D19, doi:10.1029/2002JD002520, 2003.

Michalsky, J. J., Liljegren, J. C., and Harrison, L. C.: A comparison of Sun photometer derivations of total column water vapor and ozone to standard measures of same at the Southern Great Plains Atmospheric Radiation Measurement site, J. Geophys. Res., 100(D12), 25 995-26003, 1995.

Mlawer, E. J., Clough, S. C., and Tobin, D. C.: The MT_CKD Water vapour Continuum: A Revised Perspective Including Collision Induced Effects, 11th ASSFTS Workshop, Bad Wildbad, Germany (available from http://www-imk.fzk.de/asf/ame/ ClosedProjects/assfts/O_I_7_Clough_SA.pdf), 2003.

Morland, J., Deuber, B., Feist, D. G., Martin, L., Nyeki, S., Kämpfer, N., Mätzler, C., Jeannet, P., and Vuilleumier, L.: The STARTWAVE atmospheric database, Atmos. Chem. Phys., 6, 125, 2006, http://www.atmos-chem-phys.net/6/1/2006/.

Randel, D. L., Vonder Haar, T. H., Ringerud, M. A., Stephens, G. L., Greenwald, T. J., and Combs, C. L.: A new global water vapor dataset, Bull. Amer. Meteorol. Soc., 77, 1233-1246, 1996.

Reagan, J. A., Thomason, L. W., Herman, and B. M., Palmer, J. M.: Assessment of atmospheric limitations on the determination of the solar spectral constant from ground-based spectroradiometer measurements, IEEE Trans. Geosci. Remote Sensing, GE-24, 258-266, 1986.

Ross, R. J. and Elliott, W. P.: Tropospheric water vapor climatology and trends over North America: 1973-93, J. Climate, 9, 35613574, 1996.

Schmid, B. and Wehrli, C.: Comparison of sun photometer calibration by Langley technique and standard lamp, Appl. Optics, 34, 
4500-4512, 1995.

Schmid, B., Thome, K. J., Demoulin, P., Peter, R., Mätzler, C., and Sekler, J.: Comparison of modeled and empirical approaches for retrieving columnar water vapor from solar transmittance measurements in the $0.94 \mu \mathrm{m}$ region, J. Geophys. Res., 101(D5), 9345-9358, 1996.

Schmid, B., Spyak, P. R., Biggar, S. F., Wehrli, C., Sekler, J., Ingold, T., Mätzler, C., and Kämpfer, I.: Evaluation of the applicability of solar and lamp radiometric calibrations of a precision Sun photometer operating between 300 and $1025 \mathrm{~nm}$, Appl. Optics, 37, 3923-3941, 1998.

Schmid, B., Michalsky, J. J., Slater, D. W., Barnard, J. C., Halthore, R. N., Liljegren, J. C., Holben, B. N., Eck, T. F., Livingston, J. M., Rusell, P. B., Ingold, T., and Slutsker, I.: Comparison of columnar water-vapor measurements from solar transmittance methods, Appl. Optics, 40, 1886-1896, 2001.
Shaw, G. E.: Error analysis of multi-wavelength sun photometry, Pageoph., 114, 1-14, 1976.

Shettle, E. P. and Fenn, R. W.: Models for the aerosols of the lower atmosphere and the effects of humidity variations on their optical properties, Rep. AFGL-TR-79-0214, Air Force Geophysics Lab., Hanscom, MA, 1979.

Thome, K. J., Herman, B. M., and Reagan, J. A.: Determination of precipitable water from solar transmission, J. Appl. Meteorol., 31, 157-165, 1992. 